\title{
Vibrational origin of exchange splitting and chiral-induced spin selectivity
}

\author{
J. Fransson* \\ Department of Physics and Astronomy, Uppsala University, Box 516, 75120 Uppsala, Sweden
}

(Received 14 May 2020; revised 20 November 2020; accepted 24 November 2020; published 14 December 2020)

Electron exchange and correlations emerging from the coupling between ionic vibrations and electrons are addressed. Spin-dependent electron-phonon coupling originates from the spin-orbit interaction, and it is shown that such electron-phonon coupling introduces exchange splitting between the spin channels in the structure. By application of these results to a model for a chiral molecular structure mounted between metallic leads, the chiral-induced spin selectivity is found to become several tens of percent using experimentally feasible parameters.

DOI: 10.1103/PhysRevB.102.235416

\section{INTRODUCTION}

Chiral-induced spin selectivity is an intriguing phenomenon that, to our knowledge, rests on a foundation of structural chirality, spin-orbit interactions, and strongly nonequilibrium conditions. The effect is a measure of the response to changes in the magnetic environment coupled to the active region, and the phenomenology refers back to the experimental observations of substantial changes in the charge current amplitude through chiral molecules upon changes in the external magnetic conditions [1,2]. Chiral-induced spin selectivity has been shown to not be limited to multistranded helical structures, such as double-stranded DNA molecules [3] and bacteriorhodopsin [4], but has also been observed in, for example, various types of peptides [5-9] and polyalanines [10-12] and, recently, also in helicene [13,14].

On the theoretical side, different approaches can be categorized into continuum models [15-23], tight-binding descriptions [24-31], ab initio simulations using, for example, density functional theory [32-34], and multiple scattering off magnetic impurities [35]. Thus far, the success of the modeling has been limited to qualitative aspects, while quantitative ones still appear to be out of reach. Moreover, a major deficit with these approaches is their reliance on unrealistic spinorbit interaction parameters. In an attempt solve this issue, a geometric contribution to the spin-orbit was considered in a recent theoretical work [36]. However, although this effect provides a significant contribution to the spin-orbit interaction, its existence is limited to coupled structures such as double-stranded helices/DNA, for which reason it is questionable whether this mechanism can be considered as a general

\footnotetext{
*Jonas.fransson@physics.uu.se
}

Published by the American Physical Society under the terms of the Creative Commons Attribution 4.0 International license. Further distribution of this work must maintain attribution to the author(s) and the published article's title, journal citation, and DOI. Funded by Bibsam. explanation for chiral-induced spin selectivity. It is worth noticing that previous theoretical descriptions also have successfully addressed coupled helices, although, nonetheless, conjecturing that single-stranded helices should not pertain to chiral-induced spin selectivity $[25,26]$. The mechanisms and model proposed in Refs. [25,26,36] thereby provide ways to distinguish between chiral structures that should have a viable spin selectivity, in this context, between single- and double-stranded DNA. However, the fact that the effect has been demonstrated in double- as well as single-stranded structures other than DNA structures suggests that these simple mechanisms are not likely to be sufficient for complete comprehension of chiral-induced spin selectivity. The source of the failures with these and other established theoretical approaches thus far is likely to be found in the single-electron nature of all these models.

Efforts to include electron correlations have been limited to the study in Ref. [37]. There, an on-site Coulomb interaction was added on each ionic site in a molecular chain, described by a generalized form of the Kane-Mele model [38-40], including the spin-orbit interaction and adapted to a finite helical molecular structure. Using experimentally viable spin-orbit interaction parameters, it was shown that the exchange splitting between the spin channels that was introduced by the Coulomb interaction supports a chiral-induced spin selectivity of up to $10 \%$. The exchange splitting is hence a source of substantial nonequivalence between the spin channels, which is maintained under reversal of the magnetic environment.

Another source of electron correlations pertaining to molecular structures is generated by molecular vibrations, or phonons, that couple to and distort the electronic structure. The electron-phonon coupling may introduce an attractive electron-electron interaction, which can be shown by employing the canonical transformation proposed by Lang and Firsov [41]. However, most theoretical studies concerned with electron-phonon coupling omit the analogous coupling to the spin degrees of freedom of the electrons, which can be justified whenever the spin-orbit interaction is negligible. The question of whether electron-phonon interactions make a difference in solids with non-negligible spin-orbit coupling 
has been addressed in the context of ultrafast demagnetization [42], but only as a source for decoherence. Whether electron-phonon interactions are a pertinent mechanism for chiral-induced spin selectivity is, however, an open question.

The results presented in this paper show that the cooperation of electron-phonon and spin-orbit interactions generates an exchange splitting between the spin channels which is viable for chiral-induced spin selectivity. To this end, a mechanism for spin-dependent electron-phonon coupling is introduced and discussed. This mechanism is applied to a model for a general lattice structure in which the emergence of a vibrationally induced spin splitting of the electron energies is stressed. This result should open ways to address room temperature magnetism. Finally, these findings are carried over to a model for a chiral molecule [37], which is used to show strong chiral-induced spin selectivity, tens of percent, using experimentally feasible input parameters.

The remainder of this paper is organized as follows. In Sec. II, a way to understand the origin of spin-dependent electron-phonon coupling is discussed, and a few implications of this mechanism are presented in Sec. III using a simplified model that allows for transparent comprehension. The conclusions of these two sections are carried over to a tight-binding model for chiral-induced spin selectivity in Sec. IV, and the paper is summarized and concluded in Sec. V.

\section{SPIN-DEPENDENT ELECTRON-PHONON COUPLING}

First, consider the mechanism for coupling between the electron spin and the ionic vibrational degrees of freedom through the spin-orbit interaction. The single-electron Hamiltonian is in the Schrödinger picture given by

$$
H=\frac{p^{2}}{2 m_{e}}+V(\mathbf{r})+\frac{\xi}{2}(\nabla V(\mathbf{r}) \times \mathbf{p}) \cdot \boldsymbol{\sigma},
$$

where $m_{e}$ is the electron mass, $V(\mathbf{r})$ is the effective confinement potential, and $\xi=\hbar / 4 m_{e}^{2} c^{2}$. Here, the operator $\mathbf{p}$ acts on everything to its right, whereas $\nabla$ acts only on the component directly adjacent to its right, such that $\nabla V \times \mathbf{p}=(\nabla V) \times \mathbf{p}$.

The ions are located at the positions $\mathbf{r}_{m}$, such that the potential $V(\mathbf{r})=\sum_{m} V\left(\mathbf{r}-\mathbf{r}_{m}\right)$. Assuming that the ions are not fixed in space but move about their respective equilibrium positions, such that $\mathbf{r}_{m}=\mathbf{r}_{m}^{(0)}+\mathbf{Q}_{m}$, where $\mathbf{Q}_{m}=\mathbf{r}_{m}-\mathbf{r}_{m}^{(0)}$, the Taylor expansion $V(\mathbf{r})=V_{0}+\sum_{m} \mathbf{Q}_{m} \cdot \nabla V(\mathbf{r})_{\mid \mathbf{r} \rightarrow \mathbf{r}_{m}^{(0)}}+$ ... provides the coupling between the electrons and lattice vibrations. Here, $V_{0} \equiv \sum_{m} V\left(\mathbf{r}_{m}^{(0)}\right)$ represents the equilibrium potential of the static ionic positions. Effecting this expansion in the model, Eq. (1), retaining, at most, linear orders in the displacement variable $\mathbf{Q}$, results in $H=H_{0}+H_{1}$, where

$$
\begin{aligned}
H_{0}= & \frac{p^{2}}{2 m_{e}}+V_{0}+\frac{\xi}{2} \sum_{m}\left[\nabla V\left(\mathbf{r}-\mathbf{r}_{m}^{(0)}\right) \times \mathbf{p}\right] \cdot \boldsymbol{\sigma}, \\
H_{1}= & \sum_{m}\left(\mathbf{Q}_{m} \cdot \nabla V\left(\mathbf{r}-\mathbf{r}_{m}^{(0)}\right)\right. \\
& \left.+\frac{\xi}{2}\left\{\nabla\left[\mathbf{Q}_{m} \cdot \nabla V\left(\mathbf{r}-\mathbf{r}_{m}^{(0)}\right)\right] \times \mathbf{p}\right\} \cdot \boldsymbol{\sigma}\right) .
\end{aligned}
$$

The vibrational quantum operator $a_{m \mu}\left(a_{m \mu}^{\dagger}\right)$, which is introduced according to the standard definitions, annihilates (creates) a vibration at site $m$ in mode $\mu$. Then, the ionic displacement is written $\mathbf{Q}_{m}=\sum_{\mu} l_{m \mu} \boldsymbol{\epsilon}_{m \mu}\left(a_{m \mu}+a_{m \mu}^{\dagger}\right)$, where $l_{m \mu}=\sqrt{\hbar / 2 \rho \nu \omega_{m \mu}}$ defines a length scale in terms of the density of vibrations $\rho$, system volume $v$, and vibrational frequency $\omega_{m \mu}$, whereas $\boldsymbol{\epsilon}_{m \mu}$ denotes the polarization vector.

A second quantized form of $H$ is obtained by employing the expansion $c_{\sigma}(\mathbf{r}, t)=\sum_{m} c_{m \sigma}(t) \phi_{m}|\sigma\rangle / \nu$, where $\phi_{m}=$ $\phi_{m}(\mathbf{r})$ is an eigenstate of $H_{0}$. Hence, the zero Hamiltonian is given by $\mathcal{H}_{0}=\sum_{m} \psi_{m}^{\dagger} \mathbf{E}_{m} \psi_{m}$, where the spinor $\psi_{m}=$ $\left(c_{m \uparrow} c_{m \downarrow}\right)^{t}$, whereas the on-site energy spectrum is defined by

$$
\mathbf{E}_{m}=\int \phi_{m}^{*} H_{0} \phi_{m} \frac{d \mathbf{r}}{v} .
$$

In the same fashion, the electron-phonon contributions to the model $H_{1}$ can be written as

$$
\mathcal{H}_{\mathrm{e}-\mathrm{ph}}=\sum_{\substack{m m^{\prime} \\ n \mu}} \psi_{m}^{\dagger}\left(U_{m m^{\prime} n \mu}+\mathbf{J}_{m m^{\prime} n \mu} \cdot \boldsymbol{\sigma}\right) \psi_{m^{\prime}}\left(a_{n \mu}+a_{n \mu}^{\dagger}\right),
$$

where

$$
\begin{aligned}
U_{m m^{\prime} n \mu} & =l_{n \mu} \sum_{k} \int \phi_{m}^{*} \boldsymbol{\epsilon}_{n \mu} \cdot \nabla V\left(\mathbf{r}-\mathbf{r}_{k}^{(0)}\right) \phi_{m^{\prime}} \frac{d \mathbf{r}}{v}, \\
\mathbf{J}_{m m^{\prime} n \mu} & =\frac{\xi l_{n \mu}}{2} \sum_{k} \int \phi_{m}^{*} \nabla\left\{\left[\boldsymbol{\epsilon}_{n \mu} \cdot \nabla V\left(\mathbf{r}-\mathbf{r}_{k}^{(0)}\right)\right] \times \mathbf{p}\right\} \phi_{m^{\prime}} \frac{d \mathbf{r}}{v} .
\end{aligned}
$$

The derivation of $\mathcal{H}_{\mathrm{e} \text {-ph }}$ demonstrates the existence of direct coupling between the electronic spin degrees of freedom and the ionic vibrations. In general, spin-orbit interaction requires a redefinition of what good spin quantum numbers mean, i.e., $\mathcal{H}_{0}$. However, when accompanied by the electron-phonon coupling, the spin-orbit interaction also provides an origin for spin-dependent electron-phonon interactions $\mathbf{J}_{m m^{\prime} n \mu}$. Hence, the spin-orbit and electron-phonon interactions combine into a viable spin-phonon coupling [Eq. (5b)] in addition to the generic coupling $U_{m m^{\prime} n \mu}$ between charge and vibrations [see Eq. (5a)]. This background serves as justification for the succeeding discussion.

\section{EXACT RESULT IN A SIMPLE MODEL}

In order to stress a few implications emerging from the spin-dependent electron-phonon interactions, consider a dimer model. Each site $(m=1,2)$ in the dimer vibrates with the frequency $\omega_{m}$ and comprises an electron level at the energy $\varepsilon_{m}$. The electronic and vibrational degrees of freedom are coupled through the potential $\mathbf{U}_{m}=U_{m} \sigma^{0}+J_{m} \sigma^{z}$ [see Eqs. (4) and (5)], while the elastic spin-orbit coupling [third term in Eq. (2a)] and the site dependences of the electron-phonon couplings are both neglected. A phenomenological Hamiltonian model for the considered dimer can hence be written as

$$
\mathcal{H}=\sum_{m}\left(\varepsilon_{m} \psi_{m}^{\dagger} \psi_{m}+\omega_{m} a_{m}^{\dagger} a_{m}\right)+\sum_{m} \psi_{m}^{\dagger} \mathbf{U}_{m} \psi_{m}\left(a_{m}+a_{m}^{\dagger}\right) .
$$

For simplicity and without loss of generality, the ions are assumed to vibrate with the same energy, $\omega_{n}=\omega_{0}$. This model allows for a transparent derivation of an electron-phonongenerated spin polarization, considered next. 
Hence, the canonical transformation, $\tilde{\mathcal{H}}=e^{S} \mathcal{H} e^{-S}$, with the generating operator [41]

$$
S=-\sum_{m} \frac{1}{\omega_{0}} \psi_{m}^{\dagger} \mathbf{U}_{m} \psi_{m}\left(a_{m}-a_{m}^{\dagger}\right),
$$

transforms the electron and phonon operators according to

$$
\tilde{c}_{m \sigma}=c_{m \sigma} e^{U_{m \sigma}\left(a_{m}-a_{m}^{\dagger}\right) / \omega_{0}}, \quad \tilde{a}_{m}=a_{m}-\frac{1}{\omega_{0}} \psi_{m}^{\dagger} \mathbf{U}_{m} \psi_{m},
$$

respectively, where $U_{m \sigma}=U_{m}+J_{m} \sigma_{\sigma \sigma}^{z}$, such that $\tilde{n}_{m \sigma}=$ $\tilde{c}_{m \sigma}^{\dagger} \tilde{c}_{m \sigma}=c_{m \sigma}^{\dagger} c_{m \sigma}=n_{m \sigma}$. Hence, the transformed model acquires the decoupled form

$$
\tilde{\mathcal{H}}=\sum_{m}\left(\varepsilon_{m} \psi_{m}^{\dagger} \psi_{m}+\omega_{m} a_{m}^{\dagger} a_{m}\right)-\sum_{m} \frac{1}{\omega_{0}}\left(\psi_{m}^{\dagger} \mathbf{U}_{m} \psi_{m}\right)^{2}
$$

Next, it can be noticed that the interaction term $\left(\psi_{m}^{\dagger} \mathbf{U}_{m} \psi_{m}\right)^{2}$ can be partitioned into three contributions $\hat{U}_{i}$, $i=1,2,3$, where

$$
\begin{aligned}
& \hat{U}_{1}=\sum_{m}\left[\left(U_{m}^{2}+J_{m}^{2}\right) n_{m}+4 U_{m} J_{m} s_{m}^{z}\right], \\
& \hat{U}_{2}=2 \sum_{m}\left(U_{m}^{2}-J_{m}^{2}\right) n_{m \uparrow} n_{m \downarrow}, \\
& \hat{U}_{3}=2 U_{1} U_{2} n_{1} n_{2}+4\left(U_{1} J_{2} n_{1} s_{2}^{z}+J_{1} U_{2} s_{1}^{z} n_{2}\right)+8 J_{1} J_{2} s_{1}^{z} s_{2}^{z},
\end{aligned}
$$

where $n_{m}=\psi_{m}^{\dagger} \psi_{m}$ and $s_{m}^{z}=\psi_{m}^{\dagger} \sigma^{z} \psi_{m} / 2$. Hence, writing $\tilde{\varepsilon}_{m}=\varepsilon_{m}-\left(U_{m}^{2}+J_{m}^{2}\right) / \omega_{0}$, the transformed model can be written as

$$
\begin{aligned}
\tilde{\mathcal{H}}= & \sum_{m}\left(\tilde{\varepsilon}_{m} n_{m}-4 \frac{J_{m} U_{m}}{\omega_{0}} s_{m}^{z}-2 \frac{U_{m}^{2}-J_{m}^{2}}{\omega_{0}} n_{m \uparrow} n_{m \downarrow}+\omega_{0} a_{m}^{\dagger} a_{m}\right) \\
& -\frac{2}{\omega_{0}}\left(U_{1} U_{2} n_{1} n_{2}-2\left(U_{1} J_{2} n_{1} s_{2}^{z}+U_{2} J_{1} n_{2} s_{1}^{z}\right)-4 J_{1} J_{2} s_{1}^{z} s_{2}^{z}\right) .
\end{aligned}
$$

The important observations to be made in Eqs. (10) and (11) are that the electron-phonon coupling has been converted into (i) renormalized and spin-dependent singleelectron energy [Eq. (10a)] and (ii) on-site and intersite charging and exchange interactions [Eqs. (10b) and (10c), respectively]. Hence, the one-electron states $|m \sigma\rangle=c_{m \sigma}^{\dagger}|\rangle$ are associated with the spin-dependent energies $E_{m \sigma}=$ $\tilde{\varepsilon}_{m}-2 U_{m} J_{m} \sigma_{\sigma \sigma}^{z} / \omega_{0}$, whereas the two-electron states $\left|\sigma \sigma^{\prime}\right\rangle=$ $c_{2 \sigma^{\prime}}^{\dagger} c_{1 \sigma}^{\dagger}|\rangle$ are associated with the energies

$$
\begin{aligned}
& E_{\sigma \sigma}=\sum_{m}\left(\varepsilon_{m}-\frac{U_{m \sigma}^{2}}{\omega_{0}}\right)-\frac{2}{\omega_{0}} U_{1 \bar{\sigma}} U_{2 \bar{\sigma}}+\frac{4}{\omega_{0}} J_{1} J_{2}, \\
& E_{\uparrow \downarrow}=\sum_{m} \varepsilon_{m}-\frac{U_{1 \uparrow}^{2}+U_{2 \downarrow}^{2}}{\omega_{0}}-\frac{2}{\omega_{0}} U_{1 \downarrow} U_{2 \uparrow}-\frac{4}{\omega_{0}} J_{1} J_{2}, \\
& E_{\downarrow \uparrow}=E_{\uparrow \downarrow}+\frac{2}{\omega_{0}}\left(U_{1} J_{1}-U_{2} J_{2}\right)+\frac{4}{\omega_{0}}\left(U_{1} J_{2}-J_{1} U_{2}\right) .
\end{aligned}
$$

Here, the subscript $\bar{\sigma}$ indicates spin opposite to $\sigma$. The remaining two-electron states $|2 m\rangle \equiv c_{m \downarrow}^{\dagger} c_{m \uparrow}^{\dagger}|\rangle$ have energies $E_{2 m}=2 \tilde{\varepsilon}_{m}-2 U_{m \uparrow} U_{m \downarrow} / \omega_{0}$. The three-electron states acquire a spin split similar to the one-electron states, whereas the empty state and the four-electron state both are spin independent.

Here, it should be noticed that, while configurations that lead to spin-degenerate two-electron states exist, for instance, consider equivalent sites, the one- and three-electron states are spin split in general. The one-electron states, for instance, acquire an exchange splitting of the amount $4 U_{m} J_{m}$, which is finite whenever the coupling between the electrons and vibrations is spin dependent.

In a slightly more realistic model, on- and intersite electron-electron interactions should be included with associated parameters which, typically, are significantly larger than the induced parameters $U$ and $J$. In such a consideration, the effective correlation energies are therefore merely renormalized by the electron-phonon interaction. The one-electron (as well as the two- and three-electron) energies would, nonetheless, remain spin polarized, as indicated by the second contribution in Eq. (10a). It is also worth pointing out that the results are not limited to dimers but can be straightforwardly generalized to assemblies with any number of ions.

The results from the above model, Eqs. (6)-(11), emphasize that, when accompanied by spin-orbit coupling, the electron-phonon interactions should be interpreted as a source for exchange splitting between the spin channels. This result becomes especially important in the context of chiral-induced spin selectivity, where a finite exchange splitting is crucial for the emergence of a large effect when using realistic parameters [37]. The existence of the exchange, however, does not have to originate from electronic Coulomb interactions, as will be seen in the following.

\section{CHIRAL-INDUCED SPIN SELECTIVITY}

The final part of this paper is devoted to the electronphonon-induced spin selectivity in a chiral chain of ions comprising $\mathbb{M}$ sites, phenomenologically modeled as

$$
\begin{aligned}
\mathcal{H}_{\mathrm{mol}}= & \sum_{m=1}^{\mathbb{M}} \varepsilon_{m} \psi_{m}^{\dagger} \psi_{m}+\sum_{\nu=1}^{\mathbb{M}} \omega_{\nu} a_{\nu}^{\dagger} a_{\nu} \\
& -\sum_{m=1}^{\mathbb{M}-1}\left(\psi_{m}^{\dagger} \psi_{m+1}+\text { H.c. }\right)\left[t_{0}+\sum_{\nu} t_{\nu}\left(a_{v}+a_{\nu}^{\dagger}\right)\right] \\
& +\sum_{m=1}^{\mathbb{M}-2}\left(i \psi_{m}^{\dagger} \mathbf{v}_{m}^{(+)} \cdot \boldsymbol{\sigma} \psi_{m+2}+\text { H.c. }\right) \\
& \times\left[\lambda_{0}+\sum_{\nu} \lambda_{\nu}\left(a_{v}+a_{\nu}^{\dagger}\right)\right] .
\end{aligned}
$$

Here, the molecule is described by a set of single-electron levels described by $\varepsilon_{m} \psi_{m}^{\dagger} \psi_{m}$, the first term, where $\varepsilon_{m}$ denotes the level energy, distributed along the spatial coordinates $\mathbf{r}_{m}=\left(a \cos \varphi_{m}, a \sin \varphi_{m},(m-1) c /(\mathbb{M}-1)\right), \varphi_{m}=2 \pi(m-$ $1) M /(\mathbb{M}-1), m=1, \ldots, \mathbb{M}$. Here, $a$ and $c$ define the radius and length, respectively, of the helical structure, whereas $\mathbb{M}=M N$ is the total number of sites in which the product $M$ and $N$ denote the number of laps and ions per lap, respectively. Nearest-neighboring sites, the second line in $\mathcal{H}_{\text {mol }}$, interact via direct hopping, at a rate $t_{0}$, and electron-phonon-assisted 
hopping, at a rate $t_{v}$. Similarly, the spin-orbit coupling is picked up between next-nearest-neighbor sites, the last line in $\mathcal{H}_{\text {mol }}$, through processes of the type $i \psi_{m}^{\dagger} \mathbf{v}_{m}^{(s)} \cdot \boldsymbol{\sigma} \psi_{m+2 s}, s=$ \pm 1 , where $\lambda_{0}$ and $\lambda_{v}$ denote the direct and electron-phononassisted spin-orbit interaction parameters, respectively. The vector $\mathbf{v}_{m}^{(s)}=\hat{\mathbf{d}}_{m+s} \times \hat{\mathbf{d}}_{m+2 s}$ defines the chirality of the helical molecule in terms of the unit vectors $\hat{\mathbf{d}}_{m+s}=\left(\mathbf{r}_{m}-\right.$ $\left.\mathbf{r}_{m+s}\right) /\left|\mathbf{r}_{m}-\mathbf{r}_{m+s}\right|$. The electrons are coupled to the vibrational modes $\omega_{\nu}$, which are represented by the phonon operators $a_{v}$ and $a_{v}^{\dagger}$, through the rates $t_{v}$ and $\lambda_{v}$. For simplicity and without loss of generality, each site is modeled to carry a single vibrational mode that couples to the electronic structure only on site. By omitting intersite couplings, it is justified to assume that the ions vibrate with the same energy $\omega_{0}$ and that the on-site electron-phonon coupling parameters $t_{v}=t_{1}$ and $\lambda_{v}=\lambda_{1}$ for all $v$.

Here, it is worth noticing that a reasonable ratio between the direct and electron-phonon-assisted spin-orbit coupling parameters $\lambda_{1} / \lambda_{0}$ is within the current model represented by $|\mathbf{Q}| / a \sqrt{2}$, where $\mathbf{Q}$ is the average ionic displacement. This can be seen by an order of magnitude estimate of the ratio between the chirality $\mathbf{v}$ and $\boldsymbol{\nabla} \mathbf{Q} \cdot \mathbf{v}=\left(\partial_{i} v_{j}\right) Q_{j} \hat{\mathbf{e}}_{i}$, for which it can be found that $|\mathbf{v}| \sim 2 \sqrt{2} a(\mathbb{M}-1) / c$ and $|\nabla \mathbf{Q} \cdot \mathbf{v}| \sim$ $2|\mathbf{Q}|(\mathbb{M}-1) / c$ under the assumption $4 a^{2} \ll c^{2} /(\mathbb{M}-1)^{2}$. This gives the ratio $|\mathbf{Q}| / a \sqrt{2}$. In the opposite limit, that is, $c^{2} /(\mathbb{M}-1)^{2} \ll 4 a^{2}$, the corresponding estimates are $|\mathbf{v}| \sim$ $c|\mathbf{Q}| / 2 a^{2}(\mathbb{M}-1)$ and $|\nabla \mathbf{Q} \cdot \mathbf{v}| \sim \sqrt{2} c / 2 a(\mathbb{M}-1)$, which also leads to the ratio $|\mathbf{Q}| / a \sqrt{2}$. Then, for instance, the ratio $\lambda_{1} / \lambda_{0}=0.1$ corresponds to an average ionic displacement of about $\sqrt{2} / 10 \approx 0.14$ in terms of $a$. Hence, ionic displacements in the order of fractions of $a$ should be sufficient to provide electron-phonon-assisted spin-orbit interactions that are large enough to have an influence on the spin selectivity.

The transport properties are captured by mounting the molecule in the junction between a ferromagnetic lead and a simple metallic lead. Tunneling between the lead $\chi=L, R$ and the adjacent ionic site introduces a spin-resolved level broadening through the coupling parameter $\Gamma_{\sigma}^{\chi}=\Gamma^{\chi}\left(\sigma^{0}+\right.$ $p_{\chi} \sigma^{z}$ ). Here, $\left|p_{\chi}\right| \leqslant 1$ denotes the spin polarization, whereas $\Gamma^{\chi}=2 \pi \sum_{\mathbf{k} \in \chi}\left|t_{\chi}\right|^{2} \rho_{\chi}\left(\varepsilon_{\mathbf{k}}\right)$ accounts for the tunneling rate $t_{\chi}$ and the density of electron states $\rho_{\chi}\left(\varepsilon_{\mathbf{k}}\right)$ in the lead $\chi$.

We relate the properties of the electronic structure and transport to the single-electron Green's function $\mathbf{G}_{m n}(z)=$ $\left\langle\left\langle\psi_{m} \mid \psi_{n}^{\dagger}\right\rangle\right\rangle(z), \mathbf{G}_{m} \equiv \mathbf{G}_{m m}$, through the density of electron states $\rho_{m}(\omega)=i \operatorname{sp}\left[\mathbf{G}_{m m}^{>}(\omega)-\mathbf{G}_{m m}^{<}(\omega)\right] / 2 \pi$ and charge current

$$
J_{\sigma}=\frac{i e}{h} \operatorname{sp} \int \boldsymbol{\Gamma}_{\sigma}^{L}\left[f_{L}(\omega) \mathbf{G}_{1}^{>}\left(\boldsymbol{\Gamma}_{\sigma}^{L} ; \omega\right)+f_{L}(-\omega) \mathbf{G}_{1}^{<}\left(\boldsymbol{\Gamma}_{\sigma}^{L} ; \omega\right)\right] d \omega,
$$

respectively, where sp denotes the trace over spin- $1 / 2$ space and $f_{\chi}(\omega)=f\left(\omega-\mu_{\chi}\right)$ is the Fermi-Dirac distribution function at the chemical potential $\mu_{\chi}$. The notation $\mathbf{G}_{1}^{</>}\left(\boldsymbol{\Gamma}_{\sigma}^{L} ; \omega\right)$ indicates that the calculated properties depend on the spin polarization $p_{L}$ in the left lead through the coupling parameter $\boldsymbol{\Gamma}_{\sigma}^{L}$. All calculations are made using nonequilibrium Green's functions $\mathbf{G}_{m n}^{</>}(\omega)$.
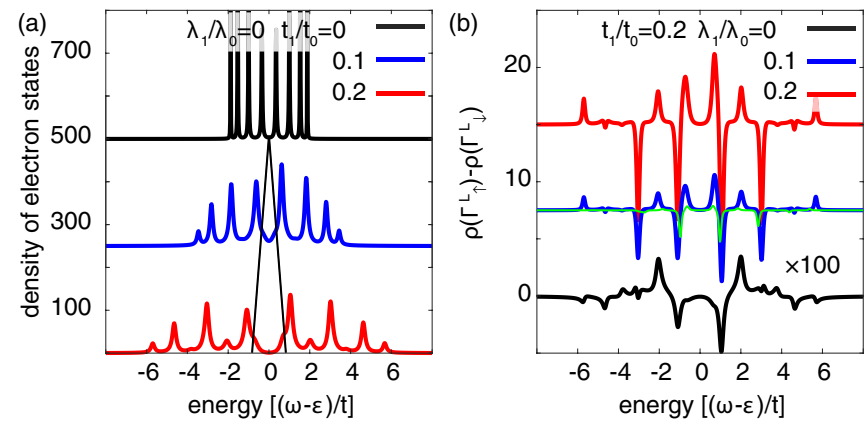

FIG. 1. (a) Density of electron states of a chiral molecule $(M \times$ $N=2 \times 4$ ) for increasing $t_{1}=0$ (black), $0.1 t_{0}$ (blue), and $0.2 t_{0}$ (red), using $\lambda_{1}=0$. (b) Difference between the density of electron states for spin polarization $\uparrow\left(p_{L}=0.5\right)$ and $\downarrow\left(p_{L}=-0.5\right)$ for $t_{1}=0.2 t_{0}$ and $\lambda_{1}=0$ (black), $0.1 \lambda_{0}$ (blue), and $0.2 \lambda_{0}$ (red). The faint green line represents the spin density of the molecule mounted between nonmagnetic leads; other parameters are as for the blue line. Other parameters are $t_{0}=40 \mathrm{meV}, \lambda_{0}=t_{0} / 40, \varepsilon-\varepsilon_{F}=-6 t_{0}, \Gamma_{0}=$ $1 / \tau_{\mathrm{ph}}=t_{0} / 4, p_{L}= \pm 0.5, p_{R}=0, \omega_{0} / t=0.010$, and $T=300 \mathrm{~K}$. An intrinsic broadening $1 / \tau_{\mathrm{ph}}=t_{0} / 4$ was used in the phonon propagation in order to smoothen the electronic densities.

The equation of motion for the Green's function $\mathbf{G}_{m n}=$ $\mathbf{G}_{m n}(z)$ can be written in the form

$$
\begin{aligned}
(z & \left.-E_{m}\right) \mathbf{G}_{m n}-\sum_{s= \pm 1}\left\{-t_{0} \mathbf{G}_{m+s n}+i \lambda_{0} \mathbf{v}_{m}^{(s)} \cdot \boldsymbol{\sigma} \mathbf{G}_{m+2 s n}\right. \\
& +\sum_{s^{\prime}= \pm 1} \boldsymbol{\Sigma}_{m}\left[t_{1}^{2} \mathbf{G}_{m+s+s^{\prime} n}-\lambda_{1}^{2} \mathbf{v}_{m}^{(s)} \cdot \boldsymbol{\sigma} \mathbf{v}_{m+2 s}^{\left(s^{\prime}\right)} \cdot \boldsymbol{\sigma} \mathbf{G}_{m+2\left(s+s^{\prime}\right) n}\right. \\
& \left.\left.-i t_{1} \lambda_{1} \boldsymbol{\sigma} \cdot\left(\mathbf{v}_{m}^{(s)} \mathbf{G}_{m+2 s+s^{\prime} n}+\mathbf{v}_{m+s}^{\left(s^{\prime}\right)} \mathbf{G}_{m+s+2 s^{\prime} n}\right)\right]\right\}=\delta_{m n} .
\end{aligned}
$$

Here, $E_{1}=\varepsilon_{1}-i \boldsymbol{\Gamma}_{\sigma}^{L} / 2, E_{m}=\varepsilon_{m}, 2 \leqslant m \leqslant \mathbb{M}-1, E_{\mathbb{M}}=$ $\varepsilon_{\mathbb{M}}-i \boldsymbol{\Gamma}^{R} / 2$, and $\mathbf{G}_{m n}=0$ for $m, n \notin\{1,2, \ldots, \mathbb{M}\}$. The self-energy $\boldsymbol{\Sigma}_{m}=\boldsymbol{\Sigma}_{m}(z)$ includes the simplest nontrivial electron-phonon interactions, given by

$$
\boldsymbol{\Sigma}_{m}(z)=\frac{n_{B}\left(\omega_{0}\right)+1-f\left(\varepsilon_{m}\right)}{z-\omega_{0}-\varepsilon_{m}}+\frac{n_{B}\left(\omega_{0}\right)+f\left(\varepsilon_{m}\right)}{z+\omega_{0}-\varepsilon_{m}},
$$

where $n_{B}(\omega)$ is the Bose-Einstein distribution function. As the exchange splitting generated by the ionic vibrations is an intrinsic property of the structure, the employed approach is justified since it captures the main effect of the electronphonon coupling. Hence, although nonequilibrium conditions may modify the exchange splitting, the gross effect of the electron-phonon interactions is captured by using the equilibrium self-energy.

\section{Numerical results}

The results from Eq. (11) indicates that a correlation gap opens under finite electron-phonon interaction. This is corroborated in the model for the chiral structure [see Fig. 1(a)], where the density of electron states for a chiral molecule $(M \times N=2 \times 4)$ is plotted for increasing spin-independent electron-phonon coupling $t_{0}$, keeping $\lambda_{1}=0$. See the caption 

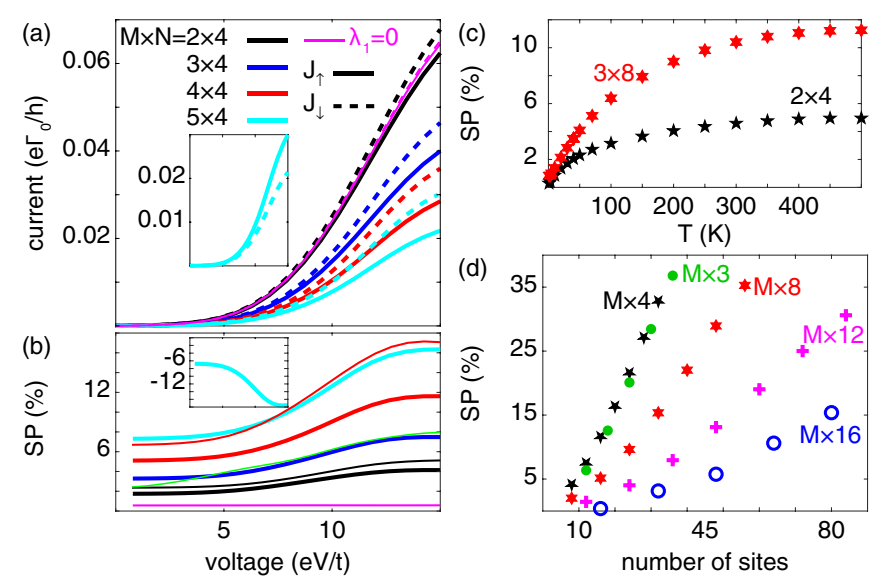

FIG. 2. (a) Charge currents and (b) corresponding spin selectivities for spin polarizations $\uparrow$ (solid lines) and $\downarrow$ (dashed lines) for structures with $2 \times 4$ (black), $3 \times 4$ (blue), $4 \times 4$ (red), and $5 \times 4$ (cyan) sites. Currents and spin selectivities in the absence of vibrations (magenta) are shown for reference. Insets show (a) the currents and (b) the spin selectivity for a $5 \times 4$ molecule with opposite chirality compared to the main panels. In (b), the faint green line represents the spin current for the molecule mounted between nonmagnetic leads; other parameters are the same as for the blue line. (c) Temperature dependence of the spin selectivity for $M \times N=2 \times 4$ and $3 \times 8$. (d) Spin selectivity at $e V / t_{0}=15$ for an increasing number of laps $M$ and an increasing number of sites per lap $N$, using $t_{1}=0.1 t_{0}$, $\lambda_{1}=0.1 \lambda_{0}$. Other parameters are as in Fig. 1. In (b), the faint lines show the spin selectivity for $2 \times 4$ (black) and $4 \times 4$ (red) molecules with electron levels normally distributed around the mean $-6 t_{0}$ and with a standard deviation of $t_{0} / 5$.

of Fig. 1 for other parameters. The opening of the correlation gap and accompanying splitting of the density into two bands are indicated by the faint lines around zero.

In the presented simulations, the vibrational mode $\omega_{0} / t_{0}=$ 0.01 , where the reciprocal of the mode, in combination with $t_{1}$, sets the energy scale for the vibrationally induced correlation gap, that is, $U_{m}^{2} / \omega_{0}$ in Eq. (11). Low-frequency modes are abundant in organic molecules, and therefore, the spectrum used should be considered realistic. Moreover, the temperature was set to $300 \mathrm{~K}$ in order to comply with experimental conditions, while the parameters $t_{0}=40 \mathrm{meV}$ and $\lambda_{0}=t_{0} / 40$ correspond to realistic numbers.

Opening a correlation gap is, however, not crucial for spin selectivity. The reason is that spin selectivity leads to a changed charge current through the structure upon switching the direction of the magnetic environment, here, the spin polarization in the left lead. However, the vibrationally generated correlation gap is not associated with broken time-reversal symmetry, which can be seen in Fig. 1(b), where the bottom trace shows that the difference of the density of electron states for the two spin polarizations $p_{L}= \pm 0.5$ is essentially negligible. The existing difference is due to higher-order coupling between vibrationally assisted tunneling $t_{1}$ and spinorbit-induced tunneling $\lambda_{0}$. The implication is, hence, that the corresponding currents are roughly equal and the generated spin selectivity is minute [see Figs. 2(a) and 2(b), faint magenta line].
Inclusion of the spin-dependent component of the electronphonon interaction leads, as suggested by Eq. (11), to a finite exchange splitting between the spin channels. Indeed, already for weak vibrationally assisted spin-orbit couplings, the asymmetry of the electronic density with respect to the spin polarization $p_{L}$ becomes enhanced by orders of magnitude [see the middle and top traces in Fig. 1(b)]. It is therefore anticipated that the spin selectivity should be sizable in the presence of coupling between the spin-orbit and electron-phonon interactions. The plots in Figs. 2(a) and 2(b) display the polarization $p_{L}$ dependence of the charge current for $\lambda_{1} / \lambda_{0}=0.1$ and the corresponding spin selectivity $\mathrm{SP}=$ $100\left|\left(J_{\uparrow}-J_{\downarrow}\right) /\left(J_{\uparrow}+J_{\downarrow}\right)\right|$ for chiral structures with $M \times 4$, $M=2,3,4,5$ sites. The results clearly demonstrate the emergence of a significant spin selectivity in the presence of finite spin-dependent electron-phonon coupling $\lambda_{1}$. The insets in Figs. 2(a) and 2(b) show, respectively, the currents and the corresponding spin selectivity of a $5 \times 4$ molecule with the opposite chirality compared to the results in the main panel. These results demonstrate that the spin selectivity also depends on whether the chirality is left- or right-handed, in agreement with experiments (see, e.g., Refs. [6,43,44]). It is, moreover, important to notice that the overall current reduces with increasing the number of sites, while the spin selectivity simultaneously increases. The latter property is expected since the number of laps in the helix tends to accumulate and hence increase the effect of the induced exchange splitting.

While the results in the main panels are simulated for molecules with all electron levels at $\varepsilon_{m}-\varepsilon_{F}=-6 t_{0}$, the effect is robust to random variations of the energy levels. This is shown by the faint lines in Fig. 2(b), which represent the spin selectivity for $2 \times 4$ (black) and $4 \times 4$ (red) molecules where the ionic electron levels $\varepsilon_{m}$ are normal distributed around the mean value $-6 t_{0}$ with a standard deviation of $t_{0} / 2$. The imposed nonresonant conditions lead to lower current amplitudes (not shown). By contrast, these conditions are not detrimental for the spin selectivity since it leads to a nonuniform distribution of the spin polarization in the molecule. Hence, a locally enhanced spin polarization tends to increase the overall effect on the spin selectivity.

It can also be noticed that finite spin densities and spin currents are generated in the molecule also without a ferromagnetic lead [see green lines in Figs. 1(b) and 2(b)], where the chiral molecule is assumed to be mounted between nonmagnetic leads. This result is consistent with the spin moment emerging in Eq. (11). While the chiral molecules should be nonmagnetic in the isolated state, it has been observed that chiral molecules, such as $\alpha$-helix polyalanine, induce Yu-Shiba-Rusinov states when disposed on the surface of superconducting $\mathrm{NbSe}_{2}$ [45]. The emergence of these resonances suggests that these chiral molecules sustain a finite spin polarization even under nonmagnetic conditions. It is well known that the existence of Yu-Shiba-Rusinov states requires the presence of a finite (superparamagnetic) spin moment [46-48]; other measurements reported neither verify nor reject the existence of a finite moment for chiral molecules. The question of intrinsic spin polarization of the chiral molecules when in contact with metals therefore remains open. 
The vibrationally assisted spin selectivity is activated by temperature, which can be seen in Fig. 2(c), in which the temperature dependence of the spin selectivity is plotted for two different molecules. The saturation at high temperatures signifies that the spin selectivity is limited by the occupation of the vibrational modes. It can be noticed that this property of increasing spin selectivity with increasing temperature can be compared with recent observations of temperature-activated ferromagnetism [49-51]. However, while the origin of the temperature activation of the ferromagnetic order observed in these examples is unknown and by no means has to be the same, it is plausible that ionic vibrations are part of the mechanism that stabilizes the ferromagnetic order.

The plots in Fig. 2(d), finally, show the dependence of the spin selectivity as a function of the product $M \times N$, indicating a weaker spin selectivity the more sites per lap there are in the helix. This is expected since the chirality vector $\mathbf{v}_{m}^{(s)} \sim \sin \varphi_{m}$, where $\varphi_{m}$ is the angle between the vectors comprising $\mathbf{v}_{m}^{(s)}$. For an increasing number of sites per lap, $\varphi_{m} \rightarrow 0$, which reflects the fact that the curvature of the helix described by the ionic coordinates approaches zero, that is, a flat line.

A weakness of the present study is the absence of Coulomb interactions as well as the equilibrium treatment of the selfenergy in Eq. (16). While the former issue may be considered (see Ref. [37]), a full treatment, including both Coulomb and vibrational interactions, becomes numerically unattractive for structures with many sites. Concerning the latter issue, while it is doable to treat the self-energy in an appropriate nonequilibrium framework, it does not change the qualitative outcome of the study and instead tends to complicate the calculations unnecessarily. By contrast, the main purpose of the present paper is to point out the importance of the exchange splitting in the context of spin selectivity and that this splitting may emerge from vibrational sources. In the model, Eq. (13), while uniform couplings $t_{1}$ and $\lambda_{1}$ between electrons and vibrations are a simplification, it is justified since the results of vibrationally induced spin polarization do not depend on the explicit construction of these interactions. It should also be mentioned that the values of, e.g., the spin-orbit interaction parameters may be on the upper end of realistically viable values. Using these values is, however, justified since the present model entails a single mechanism that is feasible in the context of chiral-induced spin selectivity, while the phenomenon itself most likely is also governed by, e.g., electron correlations, nonequilibrium, and the environment. The full picture is hence not likely to originate in a single mechanism.

\section{v. CONCLUSIONS}

In this paper it has been shown that spin-dependent electron-phonon coupling has a realistic and sound foundation at the same level as the more commonly studied coupling between the electronic charge and lattice vibrations. The presence of both spin-independent and spin-dependent electron-phonon couplings leads to vibrationally induced exchange splitting between the spin channels in the structure, a result which may open routes to addressing room temperature magnetism. These results were shown to have great importance in the theoretical modeling and comprehension of chiral-induced spin selectivity. Using experimentally realistic numbers, particularly for the spin-orbit interaction, the calculated spin selectivity reaches several tens of percent and is therefore comparable to experimental observations.

\section{ACKNOWLEDGMENTS}

The author thanks R. Naaman for constructive discussions. Support from Vetenskapsrådet, Stiftelsen Olle Engkvist Byggmästare, and Carl Tryggers Stiftelse is acknowledged.
[1] K. Ray, S. P. Ananthavel, D. H. Waldeck, and R. Naaman, Asymmetric scattering of polarized electrons by organized organic films of chiral molecules, Science 283, 814 (1999).

[2] B. Göhler, V. Hamelbeck, T. Z. Markus, M. Kettner, G. F. Hanne, Z. Vager, R. Naaman, and H. Zacharias, Spin selectivity in electron transmission through self-assembled monolayers of double-stranded DNA, Science 331, 894 (2010).

[3] Z. Xie, T. Z. Markus, S. R. Cohen, Z. Vager, R. Gutierrez, and R. Naaman, Spin specific electron conduction through DNA oligomers, Nano Lett. 11, 4652 (2011).

[4] D. Mishra, T. Z. Markus, R. Naaman, M. Kettner, B. Göhler, H. Zacharias, N. Friedman, M. Sheves, and C. Fontanesi, Spindependent electron transmission through bacteriorhodopsin embedded in purple membrane, Proc. Natl. Acad. Sci. USA 110, 14872 (2013).

[5] M. Eckshtain-Levi, E. Capua, S. Refaely-Abramson, S. Sarkar, Y. Gavrilov, S. P. Mathew, Y. Paltiel, Y. Levy, L. Kronik, and R. Naaman, Cold denaturation induces inversion of dipole and spin transfer in chiral peptide monolayers, Nat. Commun. 7, 10744 (2016).
[6] O. Ben Dor, S. Yochelis, A. Radko, K. Vankayala, E. Capua, A Capua, S.-H. Yang, L. T. Baczewski, S. S. P. Parkin, R. Naaman, and Y. Paltiel, Magnetization switching in ferromagnets by adsorbed chiral molecules without current or external magnetic field, Nat. Commun. 8, 14567 (2016).

[7] C. Fontanesi, E. Capua, Y. Paltiel, D. H. Waldeck, and R. Naaman, Spin-dependent processes measured without a permanent magnet, Adv. Mater. 30, 1707390 (2018).

[8] E. Z. B. Smolinsky, A. Neubauer, A. Kumar, S. Yochelis, E. Capua, R. Carmieli, Y. Paltiel, R. Naaman, and K. Michaeli, Electric field-controlled magnetization in GaAs/AlGaAs heterostructures-chiral organic molecules hybrids, J. Phys. Chem. Lett. 10, 1139 (2019).

[9] S. Ghosh, S. Mishra, E. Avigad, B. P. Bloom, L. T. Baczewski, S. Yochelis, Y. Paltiel, R. Naaman, and D. H. Waldeck, Effect of chiral molecules on the electron's spin wavefunction at interfaces, J. Phys. Chem. Lett. 11, 1550 (2020).

[10] O. Ben Dor, S. Yochelis, S. P. Mathew, R. Naaman, and Y. Paltiel, A chiral-based magnetic memory device without a permanent magnet, Nat. Commun. 4, 2256 (2013). 
[11] O. Ben Dor, N. Morali, S. Yochelis, L. T. Baczewski, and Y. Paltiel, Local light-induced magnetization using nanodots and chiral molecules, Nano Lett. 14, 6042 (2014).

[12] G. Koplovitz, G. Leitus, S. Ghosh, B. P. Bloom, S. Yochelis, D. Rotem, F. Vischio, M. Striccoli, E. Fanizza, R. Naaman, D. H. Waldeck, D. Porath, and Y. Paltiel, Single domain $10 \mathrm{~nm}$ ferromagnetism imprinted on superparamagnetic nanoparticles using chiral molecules, Small 15, 1804557 (2019).

[13] V. Kiran, S. P. Mathew, S. R. Cohen, I. H. Delgado, J. Lacour, and R. Naaman, Helicenes-A new class of organic spin filter, Adv. Mater. 28, 1957 (2016).

[14] M. Kettner, V. V. Maslyuk, D. Nürenberg, J. Seibel, R. Gutierrez, G. Cuniberti, K.-H. Ernst, and H. Zacharias, Chirality-dependent electron spin filtering by molecular monolayers of helicenes, J. Phys. Chem. Lett. 9, 2025 (2018).

[15] S. Yeganeh, M. A. Ratner, E. Medina, and V. Mujica, Chiral electron transport: Scattering through helical potentials, J. Chem. Phys. 131, 014707 (2009).

[16] E. Medina, F. López, M. A. Ratner, and V. Mujica, Chiral molecular films as electron polarizers and polarization modulators, Europhys. Lett. 99, 17006 (2012).

[17] S. Varela, E. Medina, F. López, and V. Mujica, Inelastic electron scattering from a helical potential: Transverse polarization and the structure factor in the single scattering approximation, J. Phys.: Condens. Matter 26, 015008 (2014).

[18] A. A. Eremko and V. M. Loktev, Spin sensitive electron transmission through helical potentials, Phys. Rev. B 88, 165409 (2013).

[19] E. Medina, L. A. González-Arraga, D. Finkelstein-Shapiro, B. Berche, and V. Mujica, Continuum model for chiral induced spin selectivity in helical molecules, J. Chem. Phys. 142, 194308 (2015).

[20] E. Díaz, A. Conteras, J. Hernández, and F. Domínguez-Adame, Effective nonlinear model for electron transport in deformable helical molecules, Phys. Rev. E 98, 052221 (2018).

[21] X. Yang, C. H. van der Wal, and B. J. van Wees, Spin-dependent electron transmission model for chiral molecules in mesoscopic devices, Phys. Rev. B 99, 024418 (2019).

[22] E. Díaz, P. Albares, P. G. Estévez, J. M. Cerveró, C. Gaul, E. Diez, and F. Domínguez-Adame, Spin dynamics in helical molecules with nonlinear interactions, New. J. Phys. 20, 043055 (2018).

[23] K. Michaeli and R. Naaman, Origin of spin-dependent tunneling through chiral molecules, J. Phys. Chem. C 123, 17043 (2019).

[24] R. Gutierrez, E. Díaz, R. Naaman, and G. Cuniberti, Spinselective transport through helical molecular systems, Phys. Rev. B 85, 081404(R) (2012).

[25] A.-M. Guo and Q.-F. Sun, Spin-Selective Transport of Electrons in DNA Double Helix, Phys. Rev. Lett. 108, 218102 (2012).

[26] A.-M. Guo and Q.-F. Sun, Spin-dependent electron transport in protein-like single-helical molecules, Proc. Natl. Acad. Sci. USA 111, 11658 (2014).

[27] D. Rai and M. Galperin, Electrically driven spin currents in DNA, J. Phys. Chem. C 117, 13730 (2013).

[28] S. Matityahu, Y. Utsumi, A. Aharony, O. Entin-Wohlman, and C. A. Balseiro, Spin-dependent transport through a chiral molecule in the presence of spin-orbit interaction and nonunitary effects, Phys. Rev. B 93, 075407 (2016).
[29] S. Varela, V. Mujica, and E. Medina, Effective spin-orbit couplings in an analytical tight-binding model of DNA: Spin filtering and chiral spin transport, Phys. Rev. B 93, 155436 (2016).

[30] S. Behnia, S. Fathizadeh, and A. Akhshani, Modeling spin selectivity in charge transfer across the DNA/gold interface, Chem. Phys. 477, 61 (2016).

[31] S. Dalum and P. Hedegård, Theory of chiral induced spin selectivity, Nano Lett. 19, 5253 (2019).

[32] V. V. Maslyuk, R. Gutierrez, A. Dianat, V. Mujica, and G. Cuniberti, Enhanced magnetoresistance in chiral molecular junctions, J. Phys. Chem. Lett. 9, 5453 (2018).

[33] E. Díaz, F. Domínguez-Adame, R. Gutierrez, G. Cuniberti, and V. Mujica, Thermal decoherence and disorder effects on chiralinduced spin selectivity, J. Phys. Chem. Lett. 9, 5753 (2018).

[34] M. S. Zöllner, S. Varela, E. Medina, V. Mujica, and C. Herrmann, Insight into the origin of chiral-induced spin selectivity from a symmetry analysis of electronic transmission, J. Chem. Theory Comput. 16, 2914 (2020).

[35] A. Ghazaryan, M. Lemeshko, and A. G. Volosniev, Spin Filtering in Multiple Scattering off Point Magnets, Commun. Phys. 3, 178 (2020).

[36] A. Shitade and E. Minamitani, Geometric Spin-Orbit Coupling and Chirality-Induced Spin Selectivity, New J. Phys. 22, 113023 (2020).

[37] J. Fransson, Chirality-induced spin selectivity: The role of electron correlations, J. Phys. Chem. Lett. 10, 7126 (2019).

[38] F. D. M. Haldane, Model for a Quantum Hall Effect without Landau Levels: Condensed-Matter Realization of the "Parity Anomaly," Phys. Rev. Lett. 61, 2015 (1988).

[39] C. L. Kane and E. J. Mele, $Z_{2}$ Topological Order and the Quantum Spin Hall Effect, Phys. Rev. Lett. 95, 146802 (2005).

[40] C. L. Kane and E. J. Mele, Quantum Spin Hall Effect in Graphene, Phys. Rev. Lett. 95, 226801 (2005).

[41] I. G. Lang and Y. A. Firsov, Kinetic theory of semiconductors with low mobility, Zh. Eksp. Teor. Fiz. 43, 1843 (1962) [JETP 16, 1301 (1962)].

[42] A. Baral, S. Vollmar, S. Kaltenborn, and H. C. Schneider, Reexamination of the Elliott-Yafet spin-relaxation mechanism, New J. Phys. 18, 023012 (2016).

[43] T. J. Zwang, S. Hürlimann, M. G. Hill, and J. K. Barton, Helixdependent spin filtering through the DNA duplex, J. Am. Chem. Soc. 138, 15551 (2016).

[44] M. Suda, Y. Thathong, V. Promarak, H. Kojima, M. Nakamura, T. Shiraogawa, M. Ehara, and H. H. Yamamoto, Light-driven molecular switch for reconfigurable spin filters, Nat. Commun. 10, 2455 (2019).

[45] H. Alpern, K. Yavilberg, T. Dvir, N. Sukenik, M. Klang, S. Yochelis, H. Cohen, E. Grosfeld, H. Steinberg, Y. Paltiel, and O. Millo, Magnetic-related state and order parameter induced in a conventional superconductor by nonmagnetic chiral molecules, Nano Lett. 19, 5167 (2019).

[46] H. Shiba, Classical spins in superconductors, Prog. Theor. Phys. 40, 435 (1968).

[47] L. Yu, Bound state in superconductors with paramagnetic impurities, Acta Phys. Sin. 21, 75 (1965).

[48] A. Rusinov, Superconductivity near a paramagnetic impurity, JETP Lett. 9, 85 (1969).

[49] B. Dhara, K. Tarafder, P. K. Jha, S. N. Panja, S. Nair, P. M. Oppeneer, and N. Ballav, Possible room-temperature ferro- 
magnetism in self-assembled ensembles of paramagnetic and diamagnetic molecular semiconductors, J. Phys. Chem. Lett. 7, 4988 (2016).

[50] B. Dhara, P. K. Jha, K. Gupta, V. K. Bind, and N. Ballav, Diamagnetic molecules exhibiting room-temperature ferromagnetism in supramolecular aggregates, J. Phys. Chem. C 121, 12159 (2017).
[51] A. K. Mondal, N. Brown, S. Mishra, P. Makam, D. Wing, S. Gilead, Y. Wiesenfeld, G. Leitus, L. J. W. Shimon, R. Carmieli, D. Ehre, G. Kamieniarz, J. Fransson, O. Hod, L. Kronik, E. Gazit, and R. Naaman, Long-range spin-selective transport in chiral metal-organic crystals with temperature-activated magnetization, ACS Nano (2020), doi: 10.1021/acsnano.0c07569. 